\title{
Absence of Branched Chain Acyl-Transferase as a Cause of Maple Syrup Urine Disease
}

\author{
D. J. Danner, N. Armstrong, S. C. Heffelfinger, E. T. Sewell, J. H. Priest, and L. J. Elsas \\ Division of Medical Genetics, Departments of Pediatrics and Biochemistry, Emory University School of Medicine, \\ Atlanta, Georgia 30322
}

\begin{abstract}
Decreased function of human mitochondrial branched chain $\alpha$ ketoacid dehydrogenase complex results in branched chain ketoacidemia or maple syrup urine disease. Activity of this multienzyme complex varies from 0 to $\sim 15 \%$ of wild type branched chain $\alpha$-ketoacid dehydrogenase complex activity within the population of homozygous affected individuals. We used the technique of Western Blotting with antibodies against purified bovine liver branched chain $\alpha$-ketoacid dehydrogenase complex to screen mitochondrial proteins from cultured human fibroblasts for immunocrossreactive proteins. This method probes the physical structure of the proteins forming this multienzyme complex. One patient with branched chain ketoacidemia lacked an immunoreactive transacylase protein. This protein catalyzes the transfer of the branched chain acyl group from the decarboxylase to reduced coenzyme $A$. Kinetic analysis of the enzyme activity in cell lysates from this patient confirmed that the complex would not utilize coenzyme $A$. Thus, we have defined a structural basis for an impaired multienzyme complex of mitochondria in man.
\end{abstract}

\section{Introduction}

Branched chain ketoacidemia or maple syrup urine disease (MSUD) $^{1}$ results from defects in the mitochondrial multienzyme complex, branched chain $\alpha$-ketoacid dehydrogenase (BCKD; 1, 2). Inheritance patterns for the human disorder follow Mendelian rules for an autosomal recessive trait (3), thereby suggesting nuclear coding for the four proteins composing the complex. Mutations affecting different regions of any of the structural genes coding for these proteins could lead to varying decreased function of the entire complex. Reported variations in the activity of BCKD and in the clinical expression among the patients and their obligate heterozygous parents support this hypothesis of mutations occurring in different regions of the complex (4-7). Some patients expressing $>2 \%$ of wild type BCKD activity exhibit increased enzyme function in response to oral loading with thiamin (8). Based on patient studies and tests with purified BCKD, we postulated that the response to this vitamin was due to an increased biological half-life of the complex when saturated with thiamin pyrophosphate (TPP) $(6,9-11)$.

Received for publication 9 May 1984 and in revised form 31 August 1984.

1. Abbreviations used in this paper: BCKD, branched chain $\alpha$-ketoacid dehydrogenase; CoA, coenzyme A; CRM, immunocrossreactive material; MSUD, maple syrup urine disease; TPP, thiamin pyrophosphate.

J. Clin. Invest.

(c) The American Society for Clinical Investigation, Inc. 0021-9738/85/03/858/03 $\$ 1.00$

Volume 75, March 1985, 858-860
Recently, we purified the bovine liver BCKD complex to homogeneity and showed it to contain four proteins (12). The E1 decarboxylase is composed of two proteins with $M_{\mathrm{r}}$ 's of 37,500 and 46,500. Only the larger subunit binds TPP in forming the binding site for the ketoacid substrate. E2 is a $52,000-$ mol wt protein and transfers the acyl group of the ketoacid from E1 to coenzyme A (CoA)SH. E3 is the 55,000mol wt flavoprotein lipoamide dehydrogenase which reoxidizes reduced lipoyl sulfur residues of E2. Polyclonal antibodies against the purified bovine liver complex recognize all the proteins of the BCKD complex and crossreact with other species and tissues including human diploid fibroblasts (13). Here we report the use of these antibodies to probe mitochondrial proteins of human fibroblasts for immunocrossreactive material (CRM). We describe, for the first time, the immunologic absence of a single protein of the BCKD complex. This absence of the structural component is confirmed by the concomitant loss of functional response for the putative missing protein.

\section{Methods}

Cell culture. Human diploid fibroblasts derived from control individuals and 29 cell lines derived from patients with MSUD were maintained in Dulbecco and Vogt medium supplemented with $15 \%$ fetal bovine serum as previously described (14). Several of these cells have been studied previously with regard to activity of BCKD and responsivity of the BCKD complex to TPP. All MSUD cells have been evaluated for BCKD activity. (Cell line 328:reference 5; cell lines 324, 325, and 527:reference 8; cell lines 1900, 1914, 1923, and 1944:reference 15).

Mitochondria isolation. Cells from 4-6 $150 \mathrm{~cm}^{2}$ flasks were removed from monolayer by treatment with $0.25 \%$ trypsin, washed into phosphate-buffered saline, and pelleted by centrifugation at $1000 \mathrm{~g}$ for 10 min to determine their wet weight. Mitochondria were isolated by differential centrifugation of broken cell homogenates in phosphatebuffered sucrose ( $20 \mathrm{mM}$ potassium phosphate, $\mathrm{pH} 7.4 ; 0.25 \mathrm{M}$ sucrose) essentially as previously described (16).

Mitochondrial proteins were resolved by electrophoresis in a $10 \%$ polyacrylamide gel in the presence of sodium dodecyl sulfate essentially as described by Laemmli (17) except that solutions contain $2 \mathrm{mM}$ EDTA. Resolved proteins were electroblotted to nitrocellulose as described by Heffelfinger et al. (13) except the blotting was allowed to continue overnight at room temperature. To detect antigenic proteins, the nitrocellulose was soaked in $0.15 \mathrm{M} \mathrm{NaCl}: 0.05 \mathrm{M}$ Tris- $\mathrm{HCl}, \mathrm{pH}$ 7.4, containing $1 \%$ gelatin (Sigma type III, Sigma Chemical Co., St. Louis, MO) and $0.1 \%$ Tween 20 for $60 \mathrm{~min}$. The nitrocellulose was

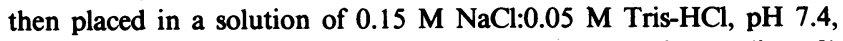
containing $0.1 \%$ Tween $20,5 \mathrm{mM}$ EDTA, $0.25 \%$ gelatin (medium 2 ) with $5 \mathrm{ml}$ of bovine serum $/ 100 \mathrm{ml}$ (total volume, $105 \mathrm{ml}$ ). After $1 \mathrm{~h}$ in this solution, $75 \mu \mathrm{l}$ of BCKD-specific rabbit immunoglobulin was added for overnight incubation at room temperature. The nitrocellulose was washed three times for $10 \mathrm{~min}$ with medium 2 and incubated with $80 \mu$ l of horseradish-peroxidase-conjugated, goat-anti(rabbit-lgG) IgG, and $1.5 \mathrm{ml}$ bovine serum in $80 \mathrm{ml}$ of medium 2 for $2 \mathrm{~h}$. Peroxidase activity was detected after four $10-\mathrm{min}$ washes of the nitrocellulose with medium 2 using a freshly prepared solution of 3,3'diaminobenzidine tetrahydrochloride (Sigma Chemical Co.; $15 \mathrm{mg}$ in 
$50 \mathrm{ml}$ of $5 \mathrm{mM}$ Tris- $\mathrm{HCl}, \mathrm{pH}$ 7.5) plus $10 \mu \mathrm{l}$ of $50 \% \mathrm{H}_{2} \mathrm{O}_{2}$. The color reaction was stopped by washing in water.

Enzyme assay. BCKD activity was determined for the fibroblast cells by quantifying ${ }^{14} \mathrm{CO}_{2}$ released from $\left[1-{ }^{14} \mathrm{C}\right]$ branched chain $\alpha$-ketoacid in the presence of cofactors as previously described (16). Partial reactions of the complex were studied by varying cofactor addition in whole cell homogenates (5).

Antibodies to the BCKD complex. Bovine liver BCKD complex was purified as described by Heffelfinger et al. (12) and used to immunize rabbits as described by Heffelfinger et al. (13).

\section{Results}

The four proteins comprising the liver BCKD complex migrate in sodium dodecyl sulfate-polyacrylamide gel electrophoresis to positions which correspond to 55,000 (E3), 52,000 (E2), $46,500(\mathrm{E} 1 \alpha)$, and $37,500 \mathrm{~mol}$ wt (E1 $\beta)$ (Fig. 1, lane 1). Based on previous data (12), it is known that these proteins comprise $<1 \%$ of the proteins forming the mitochondrion and cannot be distinguished among the total population by protein staining of the gel (Fig. 1, lane 2).

Antibodies made against the purified bovine liver complex can detect specifically the four proteins. Fig. 2 shows that when mitochondrial proteins from fibroblasts with normal BCKD activity (cell line 1271) are examined for CRM-positive BCKD proteins, a pattern identical to that seen for the purified liver complex was observed (Fig. 2, lane 2). 23 of the 24 cell lines examined with demonstrated defective BCKD activity show this four protein pattern (Fig. 2, lane 3). All proteins of the complex were immunologically present in six cell lines examined from patients with thiamin-responsive forms of branched chain ketoacidemia $(8,15)$. Cell line 328 , derived from a thiamin nonresponsive patient (5), was the only line found to lack a single antigenic protein of BCKD in the mitochondrial protein population (Fig. 2, lane 1). Only proteins for the E1 and E3 components were present in mitochondria from 328 and no other CRM was observed with altered mobility.

Since the E2 protein was apparently missing, cells from this patient should not be able to utilize CoASH in this

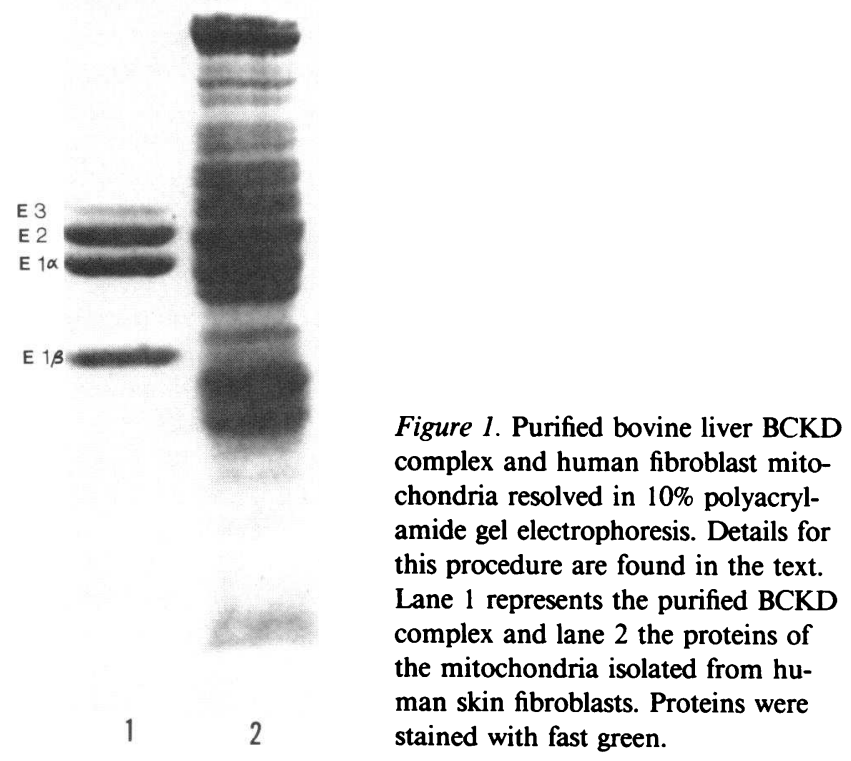

2. Immunocrossreactive proteins in mitochondria from three lines of cultured human skin fibroblasts. Details are found in the text. Lane 1 are immunoreactive products from the cells of a thiamin nonresponsive patient whose BCKD complex had $<1 \%$ control activity. Lane 2 is the mitochondrial proteins from a control

cell line with full BCKD activity and lane 3 is the mitochondrial proteins from a patient with $\sim 2 \%$ BCKD activity and represents the findings for cells from 23 patients with BCKD activity ranging from $1-15 \%$ of control cell BCKD activity.

reaction. BCKD activity in broken cell homogenates of fibroblasts is dependent upon the addition of cofactors for this reaction (5). Using these conditions of cofactor reconstitution, $\mathrm{BCKD}$ activity in this patient's cells gave a distinctive response in the presence of different combinations of cofactors. Conditions were designed to test independently the activity of E1 or E2. Fig. 3 depicts these results. Homogenates from control cells were able to decarboxylate the branched chain ketoacids in the presence of TPP/ $\mathrm{Mg}^{2+}$ alone (E1 activity) as were the homogenates from cell line 328 (Fig. 3, top). By contrast, when $\mathrm{CoASH} / \mathrm{NAD}$ was used without $\mathrm{TPP} / \mathrm{Mg}^{2+}$ (E2 activity), only control cell homogenates could decarboxylate the substrate (Fig. 3, bottom). Thus, the E2 transacylase was missing from cell line 328 by both immunologic and functional criteria.

\section{Discussion}

Over $90 \%$ of the proteins which make up the mitochondrion are coded on nuclear genes $(18,19)$. Proteins of the BCKD complex likely fall into this category since inherited defects affecting this complex in man follow Mendelian laws for transmission $(3,5)$. Three of the proteins of the BCKD complex are unique to this complex while E3, the flavoprotein lipoamide dehydrogenase, is also present in the pyruvate and $\alpha$-ketoglutarate dehydrogenase complexes (12). Currently little is known about the structural genes which code for the proteins of the BCKD complex. Our findings suggest that the genes directing the synthesis of these proteins may not be coordinately expressed. The mutation we describe has immunocompetent proteins corresponding to $\mathrm{E} 3, \mathrm{E} 1 \alpha$, and $\mathrm{E} 1 \beta$ but not for $\mathrm{E} 2$. Since the antibodies used are polyclonal for the entire complex, it is highly unlikely that all immunogenic sites are lost from

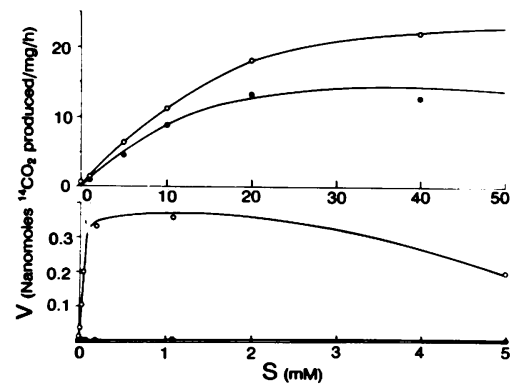

Figure 3. Ability of fibroblast cell homogenates to decarboxylate $\alpha$-keto- $\beta$-methylvalerate in the presence of two different cofactor combinations. Control cells are represented by the open symbols and the mutant no. 328 represented by the closed symbols. Top, data represented was generated with only TPP and magnesium chloride added to the assay mixture; bottom, data were obtained when coenzyme A and NAD were present without the other cofactors. 
the E2 protein and therefore not detectable by this assay. Indirect evidence for noncoordinated expression is reported from Patel's group (20). They showed that when the BCKD complex is induced by differentiation of 3T3-L1 fibroblasts into adipocytes, the proteins of BCKD increase disproportionately. E1 is increased almost 20 -fold, E2 sixfold, and E3 only fourfold based on activity measurements. Therefore, our mutant cell line will prove helpful in studying the expression and assembly of nuclear coded mitochondrial proteins.

A simplistic explanation for these findings is the lack of expression of the structural gene for E2. This could be due to the absence of the gene, lack of messenger RNA (mRNA) formation from this gene, or formation of incompetent mRNA (21). To test these possibilities, cyclic DNA probes, which are not yet available, are necessary to test the nucleic acid structures. Another explanation would be that the protein made in the cytosol is not taken up and processed by the mitochondria. The defective protein could also have an extremely short halflife and thus not be detectable. These questions are currently being tested.

It is possible for the proteins to be present and detectable by the immunoblot and still be functionally inactive. We have not identified specifically the defective enzyme of the BCKD complex in any of the cell lines where all four proteins are immunogenically present. Although defects have been described which affect the function of the similar pyruvate dehydrogenase complex and have been demonstrated in cultured fibroblasts (i.e., reference 22), our data provide the first description of decreased function in a mitochondrial multienzyme complex resulting from a missing protein within that complex. The patient from which cell line 328 was derived died in infancy despite dietary restriction and thiamin administration within the first week of life (4). Based on previous data reported for this cell line and summarized in Fig. 3, we proposed that the defect would be in the E2 protein of BCKD complex (5). Decarboxylation of the ketoacid substrate occurred only when the cell homogenate was supplemented with $\mathrm{TPP} / \mathrm{Mg}^{2+}$ but not when CoASH/NAD were present. Since E1 proteins are present, decarboxylation of the ketoacid occurred in the presence of $\mathrm{TPP} / \mathrm{Mg}^{2+}$ but the acyl-CoA product cannot form without the activity of E2. Likewise, E1 cannot be recycled without the transfer action of E2. Absence of the E2 protein (Fig. 2) in this mutant cell line provides a molecular basis for both the nonfunction of a mutant BCKD complex and the inability to stabilize the complex with TPP saturation. Our current findings further suggest that individuals who respond with clinical improvement to thiamin therapy must have present in their cells all four proteins of the complex. Cells from all thiamin responsive patients studied showed the normal profile for antigenic BCKD proteins.

\section{Acknowledgments}

This work was supported by U. S. Public Health Service grants HD 08388 and 5T35 NS07193.

\section{References}

1. Dancis, J., M. Levitz, S. Miller, and R. G. Westall. 1959. Maple syrup urine disease. Br. Med. J. 1:91-93.

2. Menkes, J. H. 1959. Maple syrup urine disease: isolation and identification of organic acids in the urine. Pediatrics. 23:348-353.
3. Tanaka, K., and L. E. Rosenberg. 1983. Disorders of branched chain amino acid and organic acid metabolism. In The Metabolic Basis of Inherited Disease. J. B. Stanbury, J. B. Wyngaarden, D. S. Fredrickson, J. L. Goldstein, and M. S. Brown, editors. McGraw-Hill, New York. 440-473.

4. Elsas, L. J., B. A. Pask, F. B. Wheeler, D. P. Perl, and S. Trusler. 1972. Classical maple syrup urine disease: cofactor resistance. Metab. Clin. Exp. 21:929-944.

5. Elsas, L. J., J. H. Priest, F. B. Wheeler, D. J. Danner, and B. A. Pask. 1974. Maple syrup urine disease: coenzyme function and prenatal monitoring. Metab. Clin. Exp. 23:529-539.

6. Elsas, L. J., and D. J. Danner. 1982. The role of thiamin in maple syrup urine disease. Ann. NY Acad. Sci. 378:404-427.

7. Rosenberg, L. E., and C. R. Scriver. 1980. Disorders of branched chain amino acid metabolism. In Metabolic Control and Disease. P. K. Bondy and L. E. Rosenberg, editors. W. B. Saunders, Philadelphia, PA. 691-699.

8. Danner, D. J., F. B. Wheeler, S. K. Lemmon, and L. J. Elsas. 1978. In vivo and in vitro response of human branched chain $\alpha$ ketoacid dehydrogenase to thiamine and thiamine pyrophosphate. Pediatr. Res. 12:235-238.

9. Danner, D. J., S. K. Lemmon, and L. J. Elsas. 1980. Cofactor binding and stabilization of mammalian liver branched chain $\alpha$ ketoacid dehydrogenase. Arch. Biochem. Biophys. 202:23-28.

10. Danner, D. J., E. D. Davidson, and L. J. Elsas. 1975. Thiamine increases the specific activity of human liver branched chain $\alpha$-ketoacid dehydrogenase. Nature (Lond.). 254:529-530.

11. Heffelfinger, S. C., E. T. Sewell, L. J. Elsas, and D. J. Danner. 1984. Direct physical evidence for stabilization of branched chain ketoacid dehydrogenase by thiamin pyrophosphate. Am. J. Hum. Genet. 36:802-807.

12. Heffelfinger, S. C., E. T. Sewell, and D. J. Danner. 1983. Identification of specific subunits of highly purified bovine liver branched chain ketoacid dehydrogenase. Biochemistry. 22:5519-5522.

13. Heffelfinger, S. C., E. T. Sewell, and D. J. Danner. 1983. Antibodies to bovine liver branched chain 2-oxoacid dehydrogenase crossreact with this enzyme complex from other tissues and species. Biochem. J. 213:339-344.

14. Danner, D. J., and J. H. Priest. 1983. Branched chain ketoacid dehydrogenase activity and growth of normal and mutant human fibroblasts: the effect of branched chain amino acid concentration in culture medium. Biochem. Genet. 21:895-905.

15. Elsas, L. J., D. J. Danner, D. Lubitz, P. M. Fernhoff, and P. P. Dembure. 1981. Metabolic consequences of inherited defects in branched chain $\alpha$-ketoacid dehydrogenase: mechanism of thiamin action. Dev. Biochem. 18:369-382.

16. Danner, D. J., and L. J. Elsas. 1975. Subcellular distribution and cofactor function of human branched chain $\alpha$-ketoacid dehydrogenase in normal and mutant cultured skin fibroblasts. Biochem. Med. 13:7-22.

17. Laemmli, U. K. 1970. Cleavage of structural proteins during the assembly of the head of bacteriophage T4. Nature (Lond.). 227: 680-685.

18. Schatz, G. 1979. How mitochondria import proteins from the cytoplasm. FEBS Lett. 103:201-211.

19. Neupert, W., and G. Schatz. 1981. How proteins are transported into mitochondria. Trends Biochem. Sci. 6:1-4.

20. Chuang, D. T., W.-L. Hu, and M. S. Patel. 1983. Induction of the branched chain 2-oxoacid dehydrogenase complex in 3T3-L1 adipocytes during differentiation. Biochem. J. 214:177-181.

21. Su, T.-S., A. L. Beaudet, and W. E. O'Brien. 1983. Abnormal mRNA for argininosuccinate synthetase in citrullinaemia. Nature (Lond.). 301:533-534.

22. Sheu, K.-F. R., C.-W. C. Hu, and M. F. Utter. 1981. Pyruvate dehydrogenase complex activity in normal and deficient fibroblasts. $J$. Clin. Invest. 67:1463-1471. 